\title{
An overview of content-based image retrieval techniques (CBIR)
}

\author{
Narjes Fathi ${ }^{1}$, Behrouz Vaseghi ${ }^{2}$, masoud Shemshadi ${ }^{3}$ \\ ${ }_{1,2,3}$ (Department of electrical and computer engineering, Abhar branch, Islamic Azad university, Abhar, Iran)
}

\begin{abstract}
In the current situation, the image plays a vital role in any aspect of life such as commercial images, satellite images, and medical images and so on. By analyzing this data, useful information can be obtaining for future uses. Image retrieval methods are divided into two general categories: 1. text-based image retrieval. 2 content-based image retrieval (CBIR). The overall system performance in content-based image retrieval is: 1 . Features extraction 2. Measuring the similarity.
\end{abstract}

Keywords: Content-based image retrieval (CBIR, Feature extraction, Image analysis techniques.

\section{Introduction}

With rapid growth of the Internet and digital imaging industry, extensive and very large databases have been created $[1,2]$. Usually the volume of images database is so vast that manually searching on it, is very time consuming and practically impossible. Therefor the need for so much more precise image retrieval systems with good speed, is considered as an important issue. By analyzing this data, useful information can be placed in the hands of man. In general, image retrieval methods can be divided into two general categories: (1) text-based image retrieval. (2) Content based image retrieval. Sometimes a combination of these two methods can be used. In the text-based retrieval systems, the user query consists of a set of words. Images contained in the database must be labeling before use. In these systems, the concepts contained in each image in a database detects by the user and the image stores on as a set of keywords. The recovery operation then acts by using keywords and tags that have been attributed before to the images. In CBIR techniques, each image labeled or tagged based on its visual characteristics. The main visual characteristics consist of color, texture and shape and they are known as low-level features [2].

\section{Content-based image retrieval (CBIR)}

CBIR techniques were developed in the 1990s. In this way each image based on its visual features labeled. The main visual characteristics consist of color, texture and shape that are known as low-level features. Image retrieval bases on these features a set of vectors of images are available. Gabor filters are used to extract frequency information [3], histogram edge color to extract the edge information [4], and the color histogram to extract color information in color images [5] and so on [2]. CBIR focuses on the characteristics of the image that provides the ability to query and recently has been focus on the study of the image database. Features can be arranged in low and then high-categorization. Users can sample images based on these features (like pattern, color, shape, location and other characteristics) at the levels that known as low level features that are more accurately categorized [1]. Fig 1 shows Block diagram of CBIR in other words, the overall system performance content-based image retrieval shows that it performs two main tasks: 1. Features extraction 2. Similarity measurement. The effectiveness of the CBIR system, depends on the performance of the algorithm it uses to extract image features. [6]

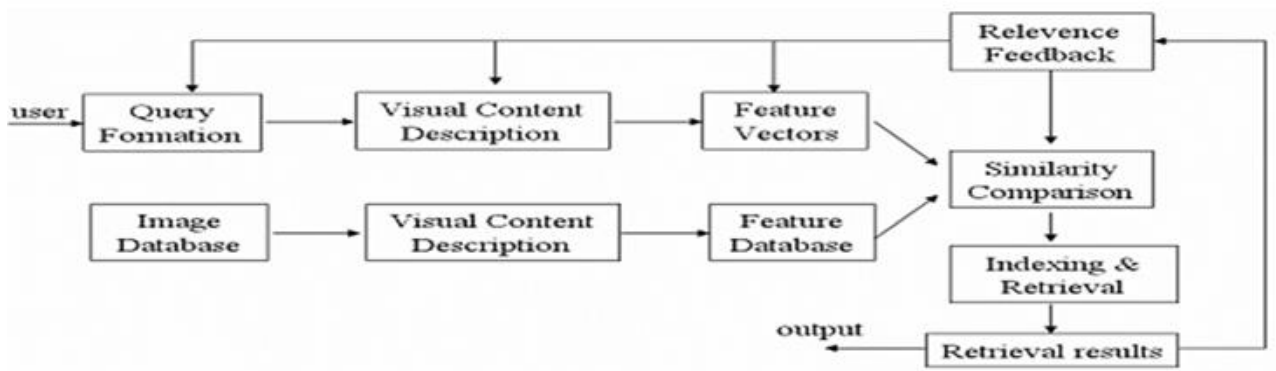

Fig. 1: Block diagram of the CBIR system [7]

III. CBIR systems performance evaluation)

The idea of performance evaluation, forecasting a CBIR system performance based on the rate of recovery. Researchers, most use the standard rate of recall and precision rate CBIR systems. Accuracy rate, and rate of relevant images retrieved reminder, related images that have been recovered are identified. If image IQ be equal to query, the rate of recall and precision rates are defined as follows: 


$$
\begin{aligned}
& \text { precision }: P\left(I_{q}\right)=\frac{\text { Count of Relevant images retrieved }}{\text { Total images retrieved }} \\
& \text { Recall }: \mathrm{R}\left(I_{q}\right)=\frac{\text { count of relevant image retrieved }}{\text { Total Relevant imagesin database }}
\end{aligned}
$$

Accuracy rate, measures the system Accuracy and reminders, of it powerfulness. The average of the benchmark for the entire image is calculated and considered as a final criterion for comparison. Average recovery rate (ARR) and the average accuracy of retrieval (ARP) are CBIR judicial systems, which mathematically, are defined as follows:

$$
\begin{aligned}
& \mathrm{ARP}=\frac{1}{|\mathrm{DB}|} \sum_{i=1}^{|D B|} P\left(I_{q}\right) \\
& \mathrm{ARR}=\frac{1}{|\mathrm{DB}|} \sum_{i=1}^{|D B|} R\left(I_{q}\right)
\end{aligned}
$$

DB is the size of the database. The top of the ARR and ARP reflects the better efficiency of CBIR. [6]

\section{Content based image retrieval techniques (CBIR)}

introduce a number of them. To find a vector feature we can describe using visual content that used locally or globally. It interest is to represent the whole picture. It is the image as a whole, while the local descriptors or low-level features represent the whole picture in the all of the area. [6] (The 1 to 7 are low-level feature extraction techniques). Finally, with regard to the recently, have used data mining techniques by researchers for image retrieval and their good results achievements, we introduced a number of these techniques as follow.

\section{- Use Local Binary Pattern (LBP):}

T.O jala and colleagues [8] introduced LBP as a black constant rotation for image indexing. LBP pixel values is calculated by comparing the gray level value of a pixel with its neighbors. LBP is better for image indexing and it used in many programs because of its gray constant index and refer all fixed rotation. They use only rotational fixed index for feature vector calculation as 10001111 that two trans from 0 to 1 or 1 to 0 takes place.

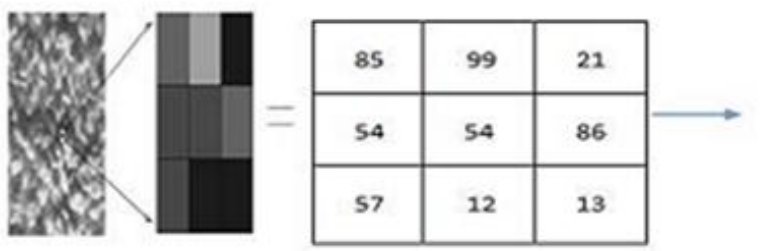

\begin{tabular}{|l|l|l|}
\hline 1 & 1 & 0 \\
\hline 1 & & 1 \\
\hline 1 & 0 & 0 \\
\hline
\end{tabular}

Binary 11001011

Decimal 203

Fig.2: LBP Calculation

Microstructures such as flat areas, lines, points, edge and histogram distribution in the norm amount of the microstructures recognized and used. It used to describe the face features because the face is a combination of Micro patterns. [6]

\section{- Use of CLBP:}

T.o jala and and et al in [8] suggest LBP that use components rather use of large component with gray level pixel differences between the center and the local pixels. Z.guo and et al in [9] suggest CLBP that uses large portion of the component to calculate the local pattern. In the LBP and CLBP, the pixel value is considered as the threshold. It is sensitive to noise and is more resistant to light.Now DP decomposed into two component parts size and symbols as shown in Fig. 4 in 3 parts. $\mathrm{N}$.shirivastara et al. [10] suggested the local structural model (LSP) that shows a large amount of pixels and the center of local disputes are considered as the threshold hence it less susceptible to noise. [6] 


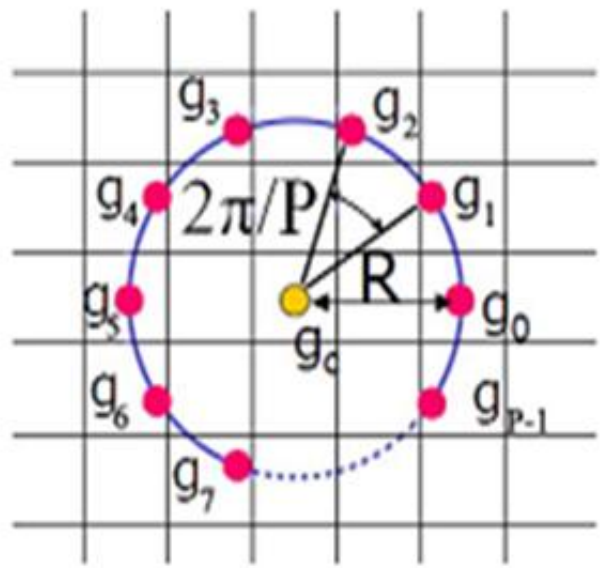

Fig.3: Central and neighboring pixels $\mathrm{P}$ with radius [9]

\begin{tabular}{|l|l|l|}
\hline 0 & 12 & 34 \\
\hline 10 & 25 & 28 \\
\hline 90 & 04 & 56 \\
\hline
\end{tabular}

(a)

\begin{tabular}{|l|l|l|}
\hline-1 & -1 & 1 \\
\hline-1 & & 1 \\
\hline 1 & 1 & 1 \\
\hline
\end{tabular}

(c)

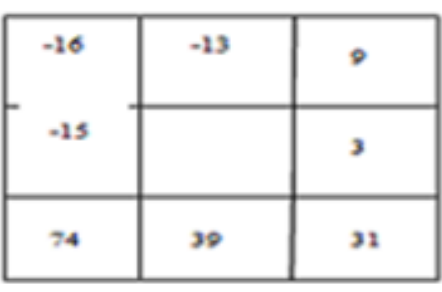

(b)

\begin{tabular}{|l|l|l|}
\hline-16 & -13 & 9 \\
\hline-15 & & 3 \\
\hline 74 & 39 & 31 \\
\hline
\end{tabular}

(d)

Fig.4: (a) block of sample $3 \times 3$; (b) loca differences; (c) the indications; and (d) a major component. [9]

\begin{tabular}{|c|c|c|}
\hline 10 & 9 & 28 \\
\hline 58 & 55 & 21 \\
\hline 32 & 83 & 59 \\
\hline
\end{tabular}

LBP: 00001011

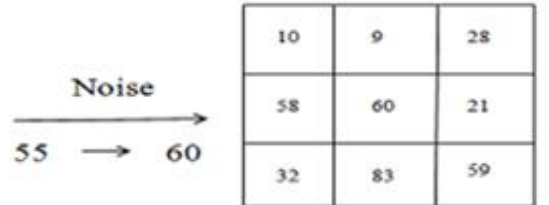

LBP: 00000010

Fig.5: LBP sensitivity to noise [11]

\section{- Using dominant LBP:}

In LBP, there must give attention to integrated patterns such as curved edges or straight edges, but some images, has a complex structure with high curved edges, corners or boundaries are crossed which makes it difficult to extract using integrated LBP [6].S.liao and et al in [12], suggest the dominant local binary patterns that uses all possible patterns rather giving consideration to mere integration patterns. Thus, complex shapes in the image are record [6].

\section{- Use a local Ternary pattern (LTP):}

Operator LBP, by producing high-resolution textures and the lighting effects, has classifying performance and itself has more resistant to light. Hence, the conversion of gray level could remain stable, but the noise in the image area almost seamlessly, is sensitive. tan and et al in [13], proposed LTP that has three code values $(-1,0,1)$, and the levels of gray in it, in an area with a width of $-\mathrm{t}$ up to $+\mathrm{t}$ around ic, which 
quantized to zero, the top and bottom, are quantized to 1 and -1 (Fig 6 ). $T$ is the threshold defined by user. The extracted features using the LTP, is powerful and stable to integrated noise in areas that LBP is not successful, but it could turn variable gray level. After calculating the triplet code, each pattern triplets, LBP is decomposed into two codes (Fig 7) [6].

\begin{tabular}{|l|l|l|}
\hline 78 & 99 & 50 \\
\hline 54 & 54 & 49 \\
\hline 57 & 12 & 31 \\
\hline Threshold \\
{$[54-\mathrm{t}, 54+\mathrm{t}], \mathrm{t}=5$}
\end{tabular}$\quad$\begin{tabular}{|l|l|l|l|}
\hline 1 & 1 & 0 \\
\hline 0 & & 0 \\
\hline 0 & -1 & -1 \\
\hline
\end{tabular}

Fig.6: Calculation of LTP [13]
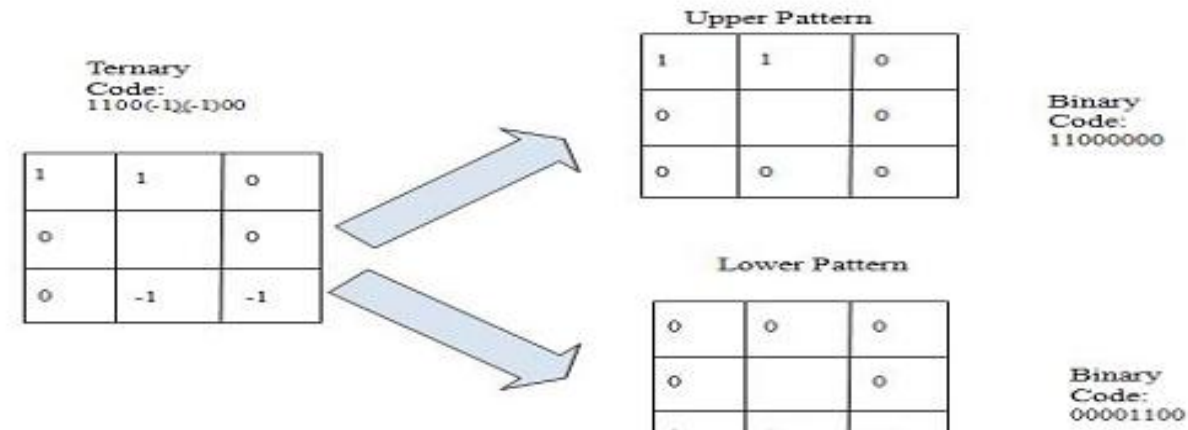

Fig.7: Analysis of LTP Code to positive and negative LBP codes. [13]

\section{- use of Local Derivative Patterns (LDP):}

Zhang [14], suggested LDP that works on directional patterns based on local derived changes, and be able to describe more detailed encoded information's which primarily binary patterns (LBP) has no such power. LDP generating a pixel image using the four directions, separately labeled, and 32-bit binary sequence produced by attaching four 8-bit binary sequences. The advantage of using local patterns of high-grade, is that, it is capable of delivering a stronger and more accurate information and greater recovery rate of LBP as well [6].

\section{- Using of local tetra patterns (LTrP):}

Murala in [15], suggests LTrP that makes relationship between the central pixel and its surrounding pixels using derivative Encoder in the horizontal and vertical directions, which results 13 template of an image. It was observed that second grade LTrP, has better resulted achievement when compared with LTrP because higher grade LTrP, is sensitive to noise.

\section{- using local mesh pattern (LMeP):}

In LBP, T.ojala in [8], encoding the relationship between the center pixel and its adjacent pixels, but in LMeP, s.murala in [16] encoding mutual relationship between adjacent using pixel data in Fig. 8, to encode them.

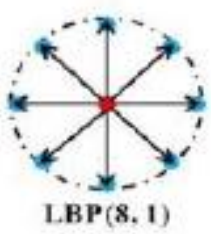

Fig.8 LBP

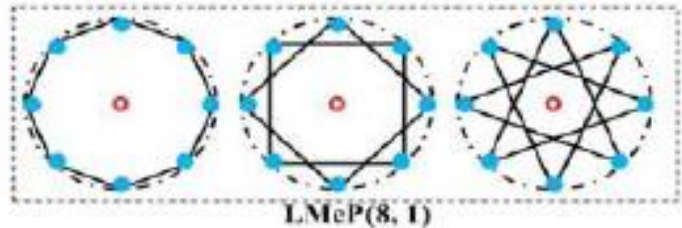

Fig.9: three LMeP calculation for given $(\mathrm{P}, \mathrm{R})$

This method, evaluate integration patterns such as LBP to reduce the computational cost. LMeP had better performance than LBP in the recovery rate when it used for biomedical images [6]. 


\section{- integration of color and pattern features derived in retrieval and indexing local picture}

S.K. Vipparthi suggested a color local derived pattern (CLDP) and an integer color local derivative pattern (ICLDP). In order to reduce the computational complexity they can use CLDP and ICLDP as uniformed patterns and uniform CLDP (CLDPU2) and ICLDP that produces uniform (ICLDPU2). The proposed method is able to exploit individual spectral channels (R, G, and B) and co-related pairs (RG, GB, BR) spectral channels of information, just extracts levels of gray or color information derived in tissue pattern. In other words, local color (CLDP), extracted texture features (LDP) are obtain by R and G and B color channels.ICLDP proposed by extracted texture features (LDP) is common spectral channels such as, RG, RB, GB, GR, BR and BG that are obtained. In this work, a neighbor of the center pixel spectral channels with surrounding spectral channels compared to the other and vice versa. In this way, in order to reduce the length of the feature vector, image ICLDP features are extracted only from the $\mathrm{O}^{0}$ and RGB color images and decomposed into different spectral channels. After checking the database with Corel 10000 and Corel 5000 a significant improvement was achieved in terms of accuracy, precision average recovery (ARP), average recall rate (ARR) in comparison with (LBP) or locally derived pattern (LDP) and other techniques, and the art of image government (state-of-the-art) shows better recovery. [17]

\section{- Conceptual model for content-based image recovery systems:}

M.srdary Zarchi have proposed a conceptual model. Visual results are similar when it used as a second layer to identify the coordinates used as a complex concept called visual composite. To identify visual composite, a structure is provided the emergence of the middle range of components together and determines their coordinates. Recognition of visual composite in this structure, do if sub-hunting filter element used and the coordinates of its range, based on the probability density function, measured so that the composite visual template parameters are used by embedded SVM. The proposed model was tested by Pascal VOC dataset. Experimental results show that the proposed model in a similar fashion, visual image retrieval based on conceptual method has been superior results (slightly higher than). [18].

\section{- Multi-Bin exhaustive-equivalent search (MBEES):}

Quantization errors of the major problems in quantization methods, are clustering and hashing. The reason is that the quantization of such patterns, can cause immediate neighbors in the bin or bucket of separation descriptors act separately, in other words, hash way, may code hash to describe the different findings from the code hash closest in neighborhood, and has incorrect results in the matching recovery, it means false positive, in Fig. 10. The purpose of the proposed method, is minimum errors in quantization, rather than using just a bin or bucket containing the descriptor query to examine the nearest bin or clusters of neighbors in order to minimize the errors quantized, in the study (Figure 10). As a result, this approach, called multi bin search. Of course, there is a balance between time and accuracy of search, because the search for more data, increased time and carefully searched.

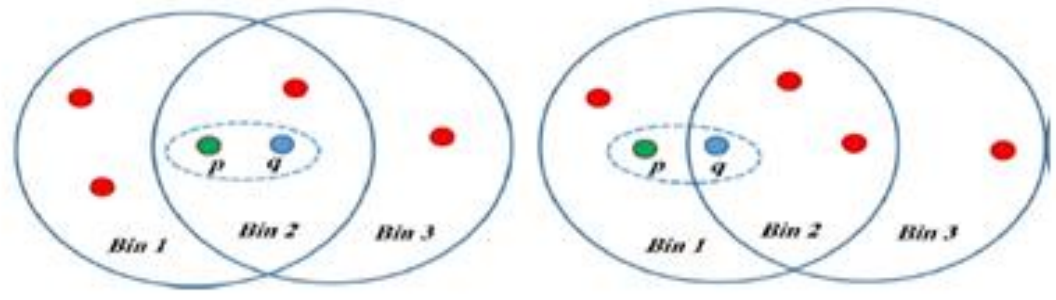

Fig.10: Left: query points $q$ and $p$ nearest neighbor in a bin or spikes. Right: the query $p$ and $p$ and its nearest neighbor in the cluster or other bin.
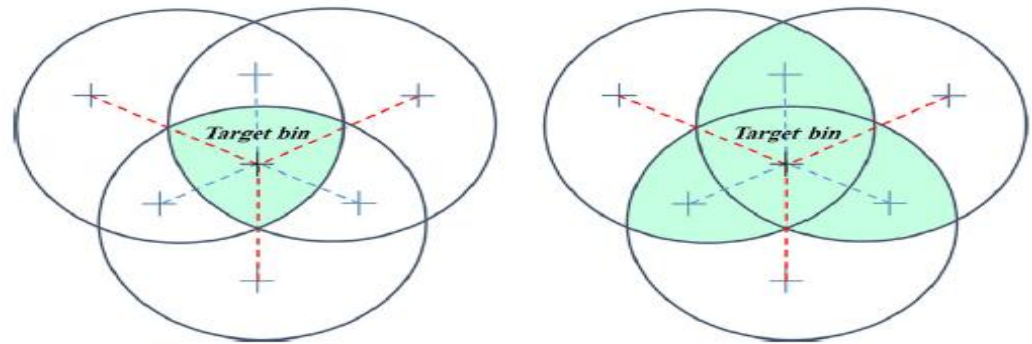

Fig.11: Left: Searching for single bin, just as aim, for parts of the search ANN (colored area). Right: Searching of multi-bin, in addition the target bin, nearest neighboring points bin are searched ANN (colored area) (Figure color in the online version is available). [19] 


\section{- Proposed Triple Criteria AL Method:}

B.Demir proposed TCAL (Active Learning with three new measures) for the development of primary education during the period of $\mathrm{RF}^{1}$ in CBIR programs offered.AL method propose objectives, as follows: achieving educational collection of images related and unrelated and the lowest number marked of occurrences of the image query that could be RF and retrieve images similar to the earlier image query with high accuracy. TCAL method proposed in the context of binary SVM-defined categories and picture category $S=\{X 1, X 2, \ldots$, $\mathrm{Xh}$ \} and ' $\mathrm{h}$ ' in each iteration of the RF selection, includes: 1) non-deterministic (ambiguous) 2) and the greatest possible diversity relation to each other and 3) It is located in the highest area feature by space histogram of the image. Images uncertainty, based on $\mathrm{MS}^{2}$ strategy is evaluated, and diversity and image density clustering based on a new strategy, could be assessed. Per Repeat proposed, AL method, the above three criteria together with a strategy based on two consecutive step is useful to choose the right images $\mathrm{S}$ for evaluation. In the first step, $\mathrm{m}>$ $\mathrm{h}$ picture is more uncertain, based on standard MS technique, and the y-elected. In the second step, the image with the most versatile $\mathrm{h} \mathrm{m}$ uncertain picture (or vague) choice, then feature of more dens areas space is selected $(\mathrm{m}>\mathrm{h}>1)[20]$.

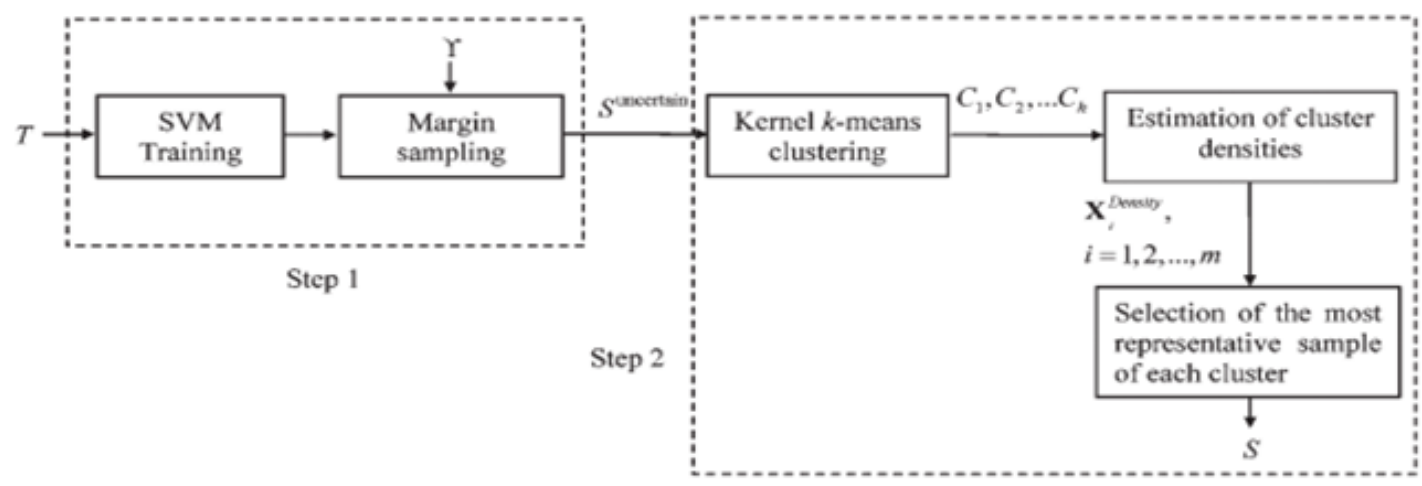

Fig.12: Block diagram of the proposed in [20]

- Image retrieval model ICTEDCT-CBIR:

Sherin m.Youssef proposed ICTEDCT-CBIR model that involves following steps (Fig 13).

1. transfer to HSV color space.

2. Apply a multiscale curve let transform to get different multiscale sub bands.

3. The proposed Region-based sub band clustering approach (RBSC). For each selected cluster i $(\mathrm{i}=1, \ldots, \mathrm{Ndcd})$, the centroid vi gravity. Based on each cluster in descending order of the number of clusters, fill in the DCD structure of Eq.

4. feature extraction using dominant color extraction (DCE)

5. feature extraction using gray-level co-occurrence matrix (GLCM), contrast, entropy, energy and inverse difference.

6. Similarities matching using similar Highest priority (MSHP) principle based on Bipartite graph of sub blocks of both query and target images [21].

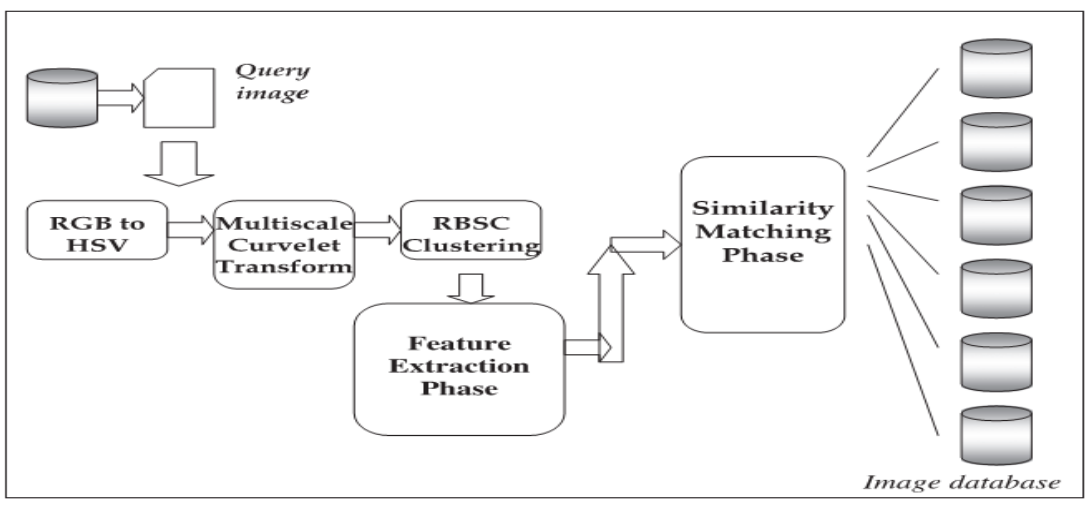

Fig.13: Model ICTEDCT-CBIR [21] 


\section{- Clustering and association rules:}

N.joseph.c proposed an approach in which the method of extraction Such as clustering and association rules used as well. Data are extracted from the image and it also integrates with several features such as visual and textual.... The results of testing the system by checked database extracts CLEF 2011. After analyzing the results respectively are, $2 \%$ to $70 \%$ that mean least support (support) and confidence (confidence). The system is based on precision and recall bas and other existing image retrieval methods, had a good result and higher performance. The following figure shows the process [22].

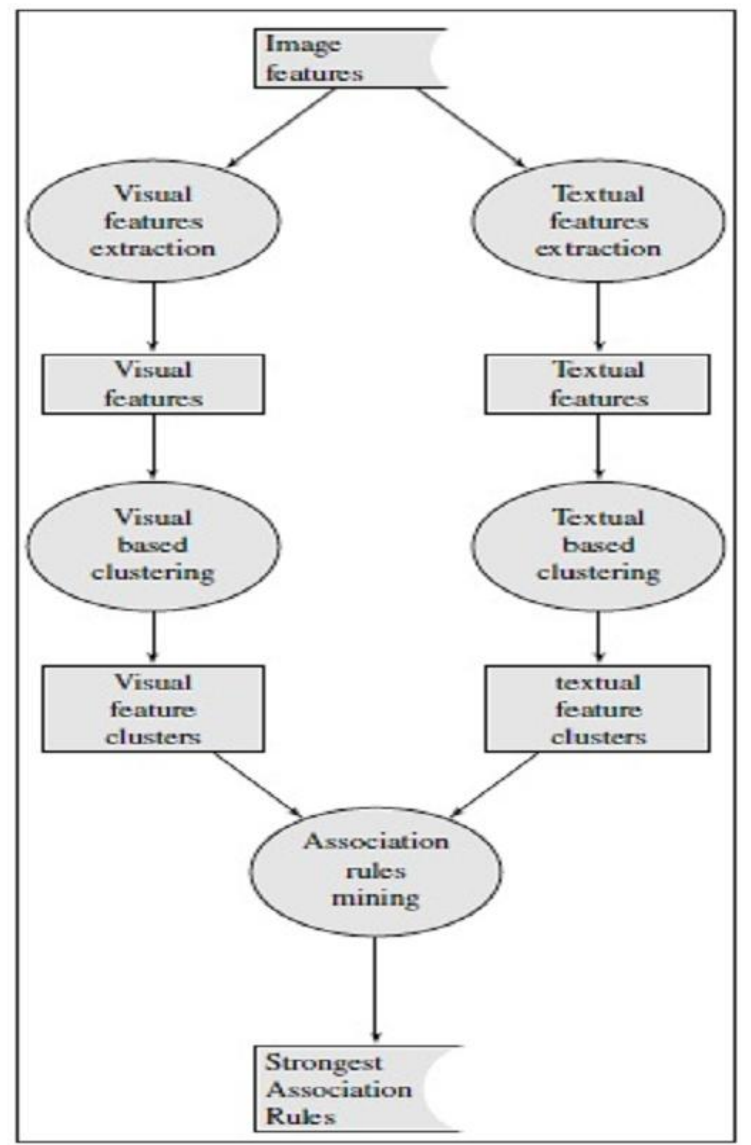

Fig.14: Data Extraction

\section{- Clusters are optimized based on image retrieval:}

A.Kannan, proposed a new technique called optimal cluster-based image retrieval, for image retrieval systems to improve user interaction, and it was introduced by taking full advantage of similar information. Index, describing the images based on color profile, with massive feature vectors, are made color sample and it is representative of the distribution. [23]

The color components RGB, are taken from each image. Then, the mean values of R and G and B for both image query (query) obtain and the target image is calculated. The three considered average values for each image, stored as its features. Then, images with a high ranking, based on the characteristics of the pattern (texture) their re-grouped. Template-based methods, parameters collect the statistics are. The statistical characteristics of gray levels, one of the most effective methods are used to classify the pattern. Gray level cooccurrence matrix (GLCM), to extract the second image is used. GLCM, has been successfully used to calculate pattern. Template parameters, such as entropy, contrast, homogeneous, standard deviation, mean and variance, Pictures query and purpose, are calculated. Based on the calculated values, the picture is needed to, mined. The pre-processed images in the database, based on factors such as MLE (maximum likelihood estimate), in the categories of low, medium pattern and large pattern, divided. Pictures are categorized by color features are extracted and retrieved, in the first phase by averaging technique such C category. Then, GLCM pattern extracted by model parameters such as contrast, correlation, mean, variance and standard deviation, are explored. Pictures query and target values, compared with Euclidean distance method.Kannan et al., Have introduced a new method for classifying image to reduce time looking up images in database images. The content of the image, in three categories, can be grouped: 
a. Accurate picture with high pattern

b. Accurate picture with average pattern

c. Accurate picture with low pattern

- $\quad$ Searching case based on annotations:

Marking or indexing system, automatically, grab information's then label collected info's. The proposed system, called large dataset. Website owners made a name consisting of 2.45 million distinct image that has 421436 names and has order of large magnitude scales over previous data sets. Collecting and labeling of such a large dataset, is a big challenge in the current multimedia search methods as well [24].

\section{Conclusion}

Since image plays a vital role in any aspect of life, Such as commercial images, Satellite, medicine and so on. The analysis of this data can be collect very useful information that can be useful for to users. CBIR In many cases, including commercial registration, medical diagnostics, crime prevention, Web search, historical research and other applications, has its importance. Extended use of CBIR in the machinery vision systems has attracted the attention of many researchers. Since content based image retrieval systems extracts features and has an important role by extraction techniques use in features extracting. Finally, recent data mining methods used in CBIR and good results have also introduced and a number of their usefulness listed in the table below that compared with each other.

Table 1: works in the field of CBIR and compared different techniques

\begin{tabular}{|c|c|c|c|c|c|}
\hline z̊ & Year & Researcher & Suggested method & The data set used & Results \\
\hline 1 & 2002 & T.ojala Et al & $\begin{array}{l}\text { Feature extraction } \\
\text { using local binary } \\
\text { pattern (LBP) }\end{array}$ & Albums Brodatz & Reduce the computational cost \\
\hline 2 & 2010 & z.guo Et al & $\begin{array}{l}\text { Full feature } \\
\text { extraction using LBP } \\
(\text { CLBP) }\end{array}$ & & $\begin{array}{l}\text { Sensitive to light, noise and resistance } \\
\text { to change. Tissue features extracted as } \\
\text { well. }\end{array}$ \\
\hline 3 & 2009 & S.liao Et al & $\begin{array}{l}\text { The dominant feature } \\
\text { extraction using LBP }\end{array}$ & $\begin{array}{l}\text { Outex 'Brodatz ' } \\
\text { Meastex and CUReT } \\
\text { database file search }\end{array}$ & $\begin{array}{l}\text { Myknd.v complex shapes in an image } \\
\text { recorded the highest accuracy in } \\
\text { classifying tissue images in different } \\
\text { situations. }\end{array}$ \\
\hline 4 & 2010 & X.tan Et al & $\begin{array}{l}\text { Feature extraction } \\
\text { using 3-local model } \\
(\text { LTP) }\end{array}$ & FRGC-204 & $\begin{array}{l}\text { Resistant to noise in integrated LBP } \\
\text { and more stable }\end{array}$ \\
\hline 5 & 2010 & B.ZhangEt al & $\begin{array}{l}\text { Feature extraction } \\
\text { using locally derived } \\
\text { model }\end{array}$ & $\begin{array}{l}\text { FERET, } \\
\text { CAS-PEAL, CMU- } \\
\text { PIE, Extended Yale B, } \\
\text { FRGC }\end{array}$ & $\begin{array}{l}\text { Capable of producing more powerful } \\
\text { and more accurate information and } \\
\text { greater recovery rate than the LBP }\end{array}$ \\
\hline 6 & 2012 & S.murala Et al & $\begin{array}{l}\text { Tetra local feature } \\
\text { extraction using 4- } \\
\text { pattern LTrP }\end{array}$ & $\begin{array}{l}\text { Corel } 1000, \\
\text { Brodatz, } \\
\text { MIT VisTex }\end{array}$ & $\begin{array}{l}\text { TrP second degree compared to higher } \\
\text { grade TrP has better results. LTrP } \\
\text { higher degree is more sensitive to } \\
\text { noise }\end{array}$ \\
\hline 7 & 2013 & S.murala Et al & $\begin{array}{lr}\text { LMP local feature } \\
\text { extraction } & \text { using } \\
\text { mesh pattern } & \\
\end{array}$ & $\begin{array}{l}\text { OASIS-MRI, } \\
\text { NEMA-CT, } \\
\text { VIA/I-ELCAP }\end{array}$ & $\begin{array}{l}\text { Reduce computational costs, better } \\
\text { recovery rate of LBP when used in } \\
\text { biomedical images. }\end{array}$ \\
\hline 8 & 2015 & $\begin{array}{l}\text { S.K.vilparthi, Et } \\
\text { al }\end{array}$ & $\begin{array}{lr}\text { Color and pattern } \\
\text { features for retrieval } \\
\text { local integration and } \\
\text { indexing images } \\
\text { derived } & \text { CLDP- } \\
\text { ICLDP } & \\
\end{array}$ & $\begin{array}{l}\text { Corel-5000, Corel- } \\
10000\end{array}$ & $\begin{array}{l}\text { In terms of accuracy, precision average } \\
\text { retrieve, recall, average recovery rate } \\
\text { improved significantly compared to } \\
\text { the LDP and LBP and other methods. }\end{array}$ \\
\hline 9 & 2015 & $\begin{array}{l}\text { M.srdary Zarchi, } \\
\text { Et al }\end{array}$ & $\begin{array}{l}\text { Identify objects and } \\
\text { visual composite }\end{array}$ & لياسكال VOC & $\begin{array}{l}\text { Has a similar fashion, the visual image } \\
\text { retrieval based on conceptual method } \\
\text { and preferable. }\end{array}$ \\
\hline 10 & 2014 & a.kamel Et al & $\begin{array}{l}\text { Multiple search } \\
\text { methods BEES }\end{array}$ & $\begin{array}{l}\text { University of } \\
\text { Kentucky } \\
\text { Benchmarking(UKB), } \\
\text { INRIA Holidays, } \\
\text { MIRFLICKR-1M }\end{array}$ & $\begin{array}{l}\text { Review the notes and the number of } \\
\text { items recovered faster than BoVW. }\end{array}$ \\
\hline 11 & 2014 & B.Demir, Et al & TCAL & $\begin{array}{l}\text { A series of aerial } \\
\text { imagery includes } 21 \\
\text { groups }\end{array}$ & $\begin{array}{l}\text { In terms of recall and precision } \\
\text { improved in compared to DC. }\end{array}$ \\
\hline 12 & 2012 & S. M. Youssef & ICTEDCT-CBIR & $\begin{array}{l}\text { The set includes } 1000 \\
\text { images in } 10 \text { semantic } \\
\text { groups }\end{array}$ & $\begin{array}{l}\text { Image recovery, precision, recall, and } \\
\text { significantly higher than other methods } \\
\text { in recovery and recovery by Gabor } \\
\text { wavelet-based filtering. }\end{array}$ \\
\hline
\end{tabular}




\begin{tabular}{|c|l|l|l|l|l|}
\hline 13 & 2010 & A.Kannan Et al & $\begin{array}{l}\text { Image retrieval based } \\
\text { on optimal cluster }\end{array}$ & - & $\begin{array}{l}\text { The search space is reduced by one- } \\
\text { third. }\end{array}$ \\
\hline 14 & 2014 & n.joseph.c, Et al & $\begin{array}{l}\text { Clustering and } \\
\text { Association Rules }\end{array}$ & $\begin{array}{l}\text { ImageCLEF 2011 } \\
\text { than other recovery methods and has } \\
\text { improved images }\end{array}$ \\
\hline 15 & 2014 & D. Wang, Et al & $\begin{array}{l}\text { Search on } \\
\text { annotations for (UR) }\end{array}$ & DB1000 & Shorter running time and less loss \\
\hline
\end{tabular}

\section{References}

[1]. A.Kannan, V. Mohan, and N. Anbazhagan, An Effective Method of Image Retrieval using Image Mining Techniques. arXiv preprint arXiv: 1012.0223, 2010.

[2]. S.Hayaty, S. Saryazdi, H.Nezam Abadi and A.Shamsy, new approach to indexing images based on image analysis

[3]. D.K.Park, Y.S.Jeon and C.S.Won, "Efficient use of local edge histogram descriptor", ACM workshops, 1-58113-311-1, 2000

[4]. W.Y. Ma, B.Manjunath, "Netra:a toolbox for navigation large image databases,", Proceedings of the IEEE International Conference on Image Processing, 1997, pp. 568-571.

[5]. J.Z Wang,J.Li,D. Chan,G. Wiederhold,"Semanticssensitive retrieval for digital picture libraries,” Digital Library Magazine, Vol. $5(11), 1999$

[6]. K.Juneja, et al. A survey on recent image indexing and retrieval techniques for low-level feature extraction in CBIR systems. in Computational Intelligence \& Communication Technology (CICT), 2015 IEEE International Conference on. 2015. IEEE.

[7]. F. Long, H. Zhang and D. D. Feng, "Fundamentals of Contentbased Image retrieval".

[8]. T.Ojala, M. Pietikäinen, and T. Mäenpää, Multiresolution gray-scale and rotation invariant texture classification with local binary patterns. Pattern Analysis and Machine Intelligence, IEEE Transactions on, 2002. 24(7): p. 971-987.

[9]. Z.Guo, L. Zhang, and D. Zhang, A completed modeling of local binary pattern operator for texture classification. Image Processing, IEEE Transactions on, 2010. 19(6): p. 1657-1663.

[10]. N. Shrivastava, and V. Tyagi, "An effective scheme for image texture classification based on binary local structure pattern", published in Springer, 2013, doi: 10.1007/s00371-013-0887-0.

[11]. T.Ahonen, A. Hadid, and M. Pietikainen, Face description with local binary patterns: Application to face recognition. Pattern Analysis and Machine Intelligence, IEEE Transactions on, 2006. 28(12): p. 2037-2041.

[12]. S.Liao, M.W. Law, and A. Chung, Dominant local binary patterns for texture classification. Image Processing, IEEE Transactions on, 2009. 18(5): p. 1107-1118.

[13]. X. Tan and B. Triggs, "Enhanced local texture feature sets for face recognition under difficult lighting conditions," published in IEEE Trans. Image Process., vol. 19, no. 6, Jun. 2010, pp. 1635-1650, doi: 10.1109/TIP.2010.2042645.

[14]. B. Zhang, Y. Gao, S. Zhao, and J. Liu, "Local derivative pattern versus local binary pattern: Face recognition with higher-order local pattern descriptor," published in IEEE Transaction on Image Processing, vol. 19, no. 2, pp. 533-544, Feb. 2010, doi: 10.1109/TIP.2009.2035882.

[15]. S. Murala, R. P. Maheshwari, and R. Balasubramanian," Local Tetra Patterns: A New Feature Descriptor for Content-Based Image Retrieval," published in IEEE Transactions on Image Processing, vol. 21, no. 5, May 2012, doi: 10.1109/TIP.2012.2188809.

[16]. S. Murala and Q.M. Jonathan, "Local Mesh Patterns Versus Local Binary Patterns: Biomedical Image Indexing and Retrieval" published in IEEE Journal of Biomedical and Health Informatics, 2013, doi: 10.1109/JBHI.2013.2288522.

[17]. S.K.Vipparthi, and S.K. Nagar, Integration of color and local derivative pattern features for content-based image indexing and retrieval. Journal of The Institution of Engineers (India): Series B, 2015. 96(3): p. 251-263.

[18]. M.S.Zarchi, A. Monadjemi, and K. Jamshidi, A concept-based model for image retrieval systems. Computers \& Electrical Engineering, 2015. 46: p. 303-313.

[19]. A .Kamel, Y.B. Mahdy, and K.F. Hussain, Multi-bin search: improved large-scale content-based image retrieval. International Journal of Multimedia Information Retrieval, 2015. 4(3): p. 205-216.

[20]. B.Demir, and L. Bruzzone, A novel active learning method in relevance feedback for content-based remote sensing image retrieval. Geoscience and Remote Sensing, IEEE Transactions on, 2015. 53(5): p. 2323-2334 .Machine Intelligence, IEEE Transactions on, 2002. 24(7): p. 971-987.

[21]. S.M.Youssef, ICTEDCT-CBIR: Integrating curvelet transform with enhanced dominant colors extraction and texture analysis for efficient content-based image retrieval. Computers \& Electrical Engineering, 2012. 38(5): p. 1358-1376.

[22]. C.N. Joseph, and A. Wilson .Retrieval of images using data mining techniques. in Contemporary Computing and Informatics (IC3I), 2014 International Conference on. 2014. IEEE.

[23]. A.Khodaskar, and S. Ladhake. Image mining: an overview of current research. in Communication Systems and Network Technologies) CSNT), 2014 Fourth International Conference on. 2014. IEEE.

[24]. D.Wang, et al ,.Mining weakly labeled web facial images for search-based face annotation. Knowledge and Data Engineering, IEEE Transactions on, 2014. 26(1): p. 166-179. 